\title{
Comparative Study of Different Space-Time Coding Schemes for MC-CDMA Systems
}

\author{
Lokesh Kumar Bansal ${ }^{1}$, Aditya Trivedi ${ }^{2}$ \\ ${ }^{1}$ Department of ECE, Nikhil Institute of Engineering and Management, Mathura, India \\ ${ }^{2}$ Department of Information and Communication Technology, ABV-Indian Institute of Information Technology and \\ Management, Gwalior, India \\ Email:lokesh_bansal@rediffmail.com,atrivedi@iiitm.ac.in \\ Received January 5, 2010; revised February 9, 2010; accepted March 10, 2010
}

\begin{abstract}
In this paper, performance of space-time trellis-code (STTC), space-time block code (STBC), and space-time trellis-code concatenated with space-time block code (STTC-STBC) for multi-carrier code-division multiple-access (MC-CDMA) system are studied. These schemes are considered by employing different detection techniques with various multi input multi output (MIMO) antenna diversity for different number of states in multi-path fading channel. The corresponding bit error rate (BER) is obtained using simulation for minimum mean-square error (MMSE), maximum-ratio combining (MRC), and equal-gain combining (EGC) receivers employing Viterbi decoder. The simulation results show that the STTC-STBC MC-CDMA system perform better compared to other schemes considered in this paper using MMSE detection and it is also observed that the performance can also be enhanced by increasing diversity using more transmitter and receiver antennas. However, this improvement in performance comes at the cost of increased computational complexity, which is calculated for different transmitting and receiving antennas.
\end{abstract}

Keywords: Space-Time Code, Space-Time Trellis-Code (STTC), Space-Time Block-Code (STBC), MIMO, MMSE, Multi-Path Channel

\section{Introduction}

Cellular services are now being used every day by millions of people worldwide and the demand is increasing exponentially. Also, there is a demand for integration of a variety of multimedia services namely short messaging, voice, data, and video. Consequently, the bit rate is required to vary from $1.2 \mathrm{kbps}$ for paging to several Mbps for video transmission. The data rate of the second generation is limited up to $9.6 \mathrm{kbps}$ [1].

The third generation cellular systems are being designed to support wideband services like high-speed internet access, video and high quality image transmission with the same quality as the fixed networks. The third generation can provide the maximum data rate ranging from $64 \mathrm{kbps}$ for mobile users to $2 \mathrm{Mbps}$ for stationary users. The drawbacks arising in the third generation system are the limitations of data rate, capacity, inter symbol interference (ISI), and inter chip interference (ICI).

For solving the problems of third generation wireless communication systems, and fulfilling the demand of high performance and broadband internet access with high spectrum efficiency, the fourth generation systems which may employ multi-carrier code-division multiple-access (MC-CDMA) technology in combination with multi-input multi-output (MIMO) antennas are proposed [2]. In the fourth generation wireless communication systems the data rate may be as high as $1 \mathrm{Gbps}$. Space-time coding techniques may be employed in conjunction with the MC-CDMA system to achieve very high data rate $[3,4]$.

In this paper, the space-time trellis-code (STTC), space-time block code (STBC), and space-time trellis-code concatenated with space-time block code (STTC-STBC) techniques are studied and applied in multi-carrier codedivision multiple-access (MC-CDMA) systems. These techniques are considered with multi input multi output (MIMO) antenna diversity in multi-path fading channel. The bit error rate (BER) is obtained for 4,8 , and 16 states using simulation. At the receiver side minimum meansquare error (MMSE), maximum-ratio combining (MRC), and equal-gain combining (EGC) techniques are used by employing Viterbi algorithm.

The organization of the rest of this paper is as follows: in Section 2, MC-CDMA systems and the space-time 
code techniques are presented. In Section 3, the mathematical representation for the space-time trellis code (STTC) MC-CDMA system is described. In Section 4, the STTC concatenated with STBC (STTC-STBC) MCCDMA system is discussed. The MMSE detector is discussed briefly in Section 5. Simulation of the error rate performance of STTC-MC-CDMA systems, STBC- MCCDMA systems, and STTC-STBC MC-CDMA systems for different states with computational complexity is carried out in Section 6. The conclusions are presented in Section 7.

\section{MC-CDMA and Space-Time Code Systems}

Multi-carrier modulation is being proposed for fourth generation wireless communication systems for high data rate application to reduce the effect of ISI. These systems solve the ISI problem by transmitting the same data symbol over a large number of narrow band orthogonal carriers [5].

An MC-CDMA signal is composed of $N$ narrowband sub-carrier signals each with symbol duration, $T_{b}$, much larger than the delay spread of the channel, $T_{d}$, hence MC-CDMA signal does not experience significant ISI. Multiple accesses is achieved with different users transmitting at the same set of sub-carriers but with spreading codes that are different to the codes of other users.

Initially, the data stream is serial to parallel converted to a number of lower rate streams. Each stream feeds a number of parallel streams with the same rate. On each of the parallel streams, bits are interleaved and spread by a PN code with a suitable chip rate. Then, these streams modulate different orthogonal carriers with a successively overlapping bandwidth [6,7].

In recent years, antenna systems which employ multiple antennas at both the base station (BS) and mobile station (MS), have been proposed and demonstrated to significantly increase system performance as well as capacity [8]. The merit of using multiple antennas or space diversity is that no bandwidth expansion or increase in transmitted power is required for capacity and performance improvements.

Space-time (S-T) coding is a technique designed for use with multiple transmits antennas. There are various approaches in coding structures, which include Alamouti space-time code (STC), space-time block code (STBC), space-time trellis code (STTC), space-time turbo trellis codes (STTTC), and layered space-time (LST) codes. STC with multiple transmit and receive antennas minimizes the effect of multi-path fading and improves the performance and capacity of digital transmission over wireless radio channels $[9,10]$.

STBC can achieve a maximum possible diversity advantage with a simple decoding algorithm. It is very at- tractive because of its simplicity. However, no coding gain can be provided by STBC. Tarokh, Seshadri, and Calderbank first introduced STTC. STTC are able to combat the effects of fading. However, STTC have a potential drawback due to the fact that its decoder complexity (maximum likelihood) grows exponentially with the number of bits per symbol [1].

\section{STTC-MC-CDMA System Model}

Trellis Coded Modulation (TCM) is a bandwidth efficient technique that combines coding and modulation, without reducing the data rate. STTC-MC-CDMA system provides coding gain and diversity. Figure 1 shows the general block diagram of space-time coded MCCDMA system. In this, the space-time trellis encoder encodes the source data; next the encoded data is interleaved, and then mapped according to the desired signal constellation. Finally, the space-time trellis encoded data symbols are modulated at each time interval, and transmitted simultaneously over different transmit antennas.

At the receiver, the received data is combined according to the different combining techniques described for MC-CDMA systems. The soft output of the combiner is sent to the deinterleaver and then finally it is applied to a space-time Trellis decoder, employing Viterbi algorithm to decode the data $[11,12]$.

At the transmitter, $K$ users transmit simultaneously the space-time trellis coded information symbols from the two transmit antennas. The frequency selective channel between transmit and receive antennas is divided into $Q$ subchannels such that each subchannel is approximately flat. For the $k$ th user, let the input message sequence $\boldsymbol{A}_{k}$, is given by,

$$
\boldsymbol{A}_{k}(n)=\left(\boldsymbol{a}_{k}(1), \boldsymbol{a}_{k}(2), \boldsymbol{a}_{k}(3), \ldots, \boldsymbol{a}_{k}(n), \ldots\right)
$$

where $\boldsymbol{a}_{k}(n)$ is a group of $m=\log _{2} M$ information bits at time $n$ and given by,

$$
\boldsymbol{a}_{k}(n)=\left(a_{k}^{1}, a_{k}^{2}, a_{k}^{3}, \ldots, a_{k}^{m}\right)
$$

The encoder maps the input stream into an $M$-ary PSK modulated signal sequence, which is given by, $\{\boldsymbol{X}(n)\}$ be the ST-Trellis encoded output. For the $k t h$ user, the output of ST-Trellis encoder, which is modulated by MC-CDMA system, is represented by the following code matrix:

$$
\boldsymbol{X}_{k}(n)=\left(\boldsymbol{x}_{k}(0), \boldsymbol{x}_{k}(1), \boldsymbol{x}_{k}(3), \ldots, \boldsymbol{x}_{k}(n), \ldots\right)
$$

where $\boldsymbol{x}_{k}(n)$ is a $n$th STT coded symbol and is given by,

$$
\boldsymbol{x}_{k}^{n_{T}}(n)=\left(x_{k}^{1}(n), x_{k}^{2}(n), \ldots, x_{k}^{n_{T}}(n)\right)^{T}
$$

The modulated signals, $x_{k}^{1}(n), x_{k}^{2}(n), \ldots, x_{k}^{n_{T}}(n)$, are 
transmitted simultaneously through $n_{T}$ transmit antennas. Here each user is assigned two distinct spreading codes to spread symbols transmitted from the two antennas. Let

and

$$
c_{k}^{1}(p)=\left[c_{k}^{1}(0), c_{k}^{1}(1), \ldots, c_{k}^{1}(p-1)\right]^{T}
$$

$$
c_{k}^{2}(p)=\left[c_{k}^{2}(0), c_{k}^{2}(1), \ldots . ., c_{k}^{2}(p-1)\right]^{T}
$$

be two spreading codes for user $k$ with processing gain $p$ which spread user $k$ 's symbols transmitted from Tx1 and Tx2, respectively, where $(.)^{T}$ denotes vector/matrix transpose. $\boldsymbol{u}_{\boldsymbol{k}}^{\boldsymbol{l}}(n)$ is defined as the signal associated with Tx1 after the spreading. Then, we have

$$
\boldsymbol{u}_{k}^{l}(n)=x_{k}^{l}(n) \boldsymbol{c}_{k}^{l}
$$

For the ST-MC-CDMA system under study, we assume that the numbers of subcarriers equal to the processing gain $p$. Each element in $\boldsymbol{u}_{\boldsymbol{k}}^{1}(n)$ is then modulated onto a sub-carrier with center frequency at $\{\omega\}_{p=0}^{p-1}$, which is common for all $K$ users. The MC-CDMA modulation can be implemented by the inverse fast Fourier transform (IFFT) (consequently, the received signal can be demodulated by FFT). Performing $p$-point IFFT on $\boldsymbol{u}_{\boldsymbol{k}}^{1}(n)$ yields

$$
\boldsymbol{z}_{\boldsymbol{k}}^{1}(n)=F^{-1} \boldsymbol{u}_{k}^{1}(n)
$$

where $F^{-1}$ denotes the $p \times p$ IFFT matrix. The contributions from all $K$ users associated with Tx 1 are given by the superposition

$$
\boldsymbol{Z}^{1}(n)=\sum_{k=1}^{K} \boldsymbol{z}_{k}^{1}(n) .
$$

Following the same procedure, we obtain the signal associated with $\mathrm{Tx} 2$ as $\boldsymbol{Z}^{2}(n)$. Both $\boldsymbol{Z}^{1}(n)$ and $\boldsymbol{Z}^{2}(n)$ are converted into a serial sequences before being transmitted through the frequency selective channels [13-15].

For the forward link, let $J$ be the number of resolvable paths. If we model the common channel between Tx1 and $\mathrm{Rx}$ for all $K$ users as a FIR filter with coefficients:

$$
\boldsymbol{h}^{1}=\left[h(0), \ldots ., h(J-1), 0_{p-J}\right]^{T}
$$

where we pad $\boldsymbol{h}^{I}$ with $p-J$ zeros to make its total length $p$, its frequency response is then given by

$$
\boldsymbol{g}^{1}=F \boldsymbol{h}^{1}=[g(0), \ldots ., g(p-1)]^{T}
$$

where $F$ denotes the $p \times p$ FFT matrix. Similarly, we define the FIR channel, also with length $J$, and its corresponding frequency response between Tx2 and Rx as $\boldsymbol{h}^{2}$ and $\boldsymbol{g}^{2}$, respectively.

$$
\begin{aligned}
& \boldsymbol{Y}(n)= \sum_{k=1}^{K}\left[\boldsymbol{\varphi}_{k}^{1} \boldsymbol{g}^{1} x_{k}^{1}(n)+\boldsymbol{\varphi}_{k}^{2} \boldsymbol{g}^{2} x_{k}^{2}(n)\right]+\boldsymbol{v}(n) \\
&= \sum_{\mathrm{k}=1}^{\mathrm{K}} \boldsymbol{C}_{k} \boldsymbol{H} \boldsymbol{x}_{k}(\mathrm{n})+\boldsymbol{v}(\mathrm{n}) \\
& \text { where } \boldsymbol{\varphi}_{k}^{l}= \operatorname{diag}\left\{\boldsymbol{c}_{k}^{1}\right\}, \boldsymbol{\varphi}_{k}^{2}=\operatorname{diag}\left\{\boldsymbol{c}_{k}^{2}\right\} \\
& \boldsymbol{C}_{k}= {\left[\begin{array}{cc}
\boldsymbol{\varphi}_{k}^{1}, \boldsymbol{\varphi}_{k}^{2}
\end{array}\right], } \\
& \boldsymbol{H}= {\left[\begin{array}{cc}
\boldsymbol{g}^{1} & 0 \\
0 & \boldsymbol{g}^{2}
\end{array}\right], } \\
& \boldsymbol{x}_{k}(n)=\left[x_{k}^{1}(n), x_{k}^{2}(n)\right]^{T}
\end{aligned}
$$

and where

$$
\boldsymbol{v}(n)=\left[v_{0}(n), \ldots ., v_{p-1}(n)\right]^{T}
$$

contains samples of the channel noise with zero-mean and variance $\sigma_{n}^{2}$. At the receiver, the received signals are first converted into parallel format and demodulated via FFT transform and then applied to deinterleaver. The interleaved data is applied to MMSE detector and then for ST-Trellis decoder for demodulation.

\section{STTC-STBC MC-CDMA System}

Space time block coding is a simple technique to achieve diversity, however there is not much significant coding gain. An outer channel code is required to yield coding gain.

Space time trellis code concatenated with space time block code (STTC-STBC) also provides coding gain. As shown in Figure 1, first the STTC encoder encodes the source data. Next, the encoded data is applied to S-T block encoder \& interleaver, and then mapped according to the desired signal constellation. Finally at each time interval, the symbols are modulated and transmitted simultaneously over different transmit antennas.

At the receiver, the received data is combined according to the combining techniques described for STBC. The soft output of the combiner is sent directly to the deinterleaver, and then finally, it is applied to a STTC decoder, such as the Viterbi algorithm, to decode the data.

\section{MMSE Detection}

In conventional MC-CDMA systems (without transmit diversity), the demodulated signals are often combined in the frequency domain in order to collect the overall received signal energy scattered on different subcarriers. Typical signal combining schemes include the maximum 


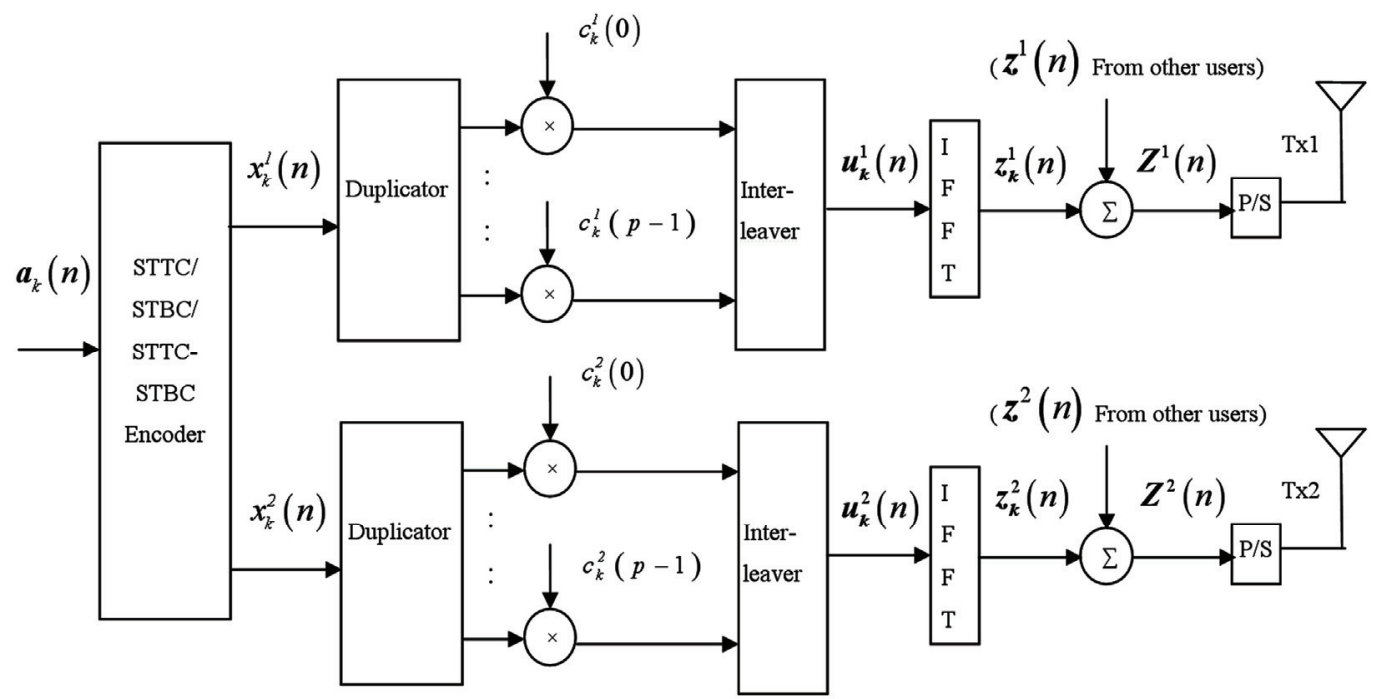

(a) Transmitter

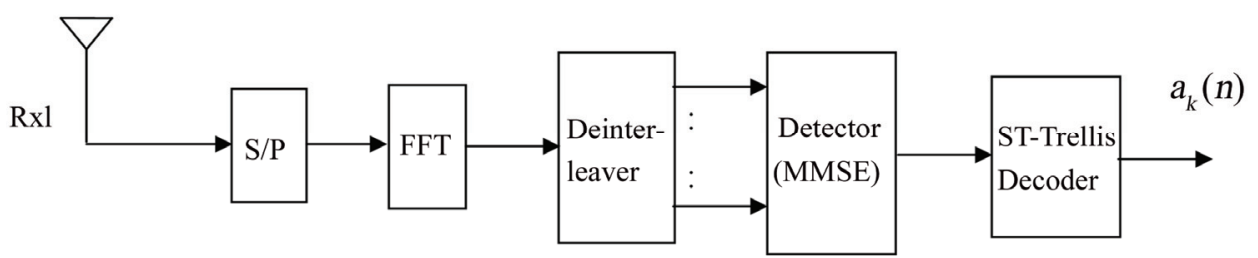

(b) Receiver

Figure 1. General block diagram of S-T coded MC-CDMA system.

ratio combining (MRC) and the equal gain combining (EGC). Both MRC and EGC are single-user detection schemes based on per-subcarrier combining, i.e. the signals at individual subcarrier are independently weighted and summed to generate decision variables. In [16], a linear multiuser minimum mean-squared error (MMSE) detector for the STC-MC-CDMA systems is presented which performs joint weighting and combining on all subcarriers by utilizing the mean-squared error (MSE) criterion. We consider the two demodulated symbols at a time. Define

$$
\begin{aligned}
\boldsymbol{G}^{1} & =\operatorname{diag}\left\{\boldsymbol{g}^{1}\right\}, \\
\Theta^{1} & =\left[\boldsymbol{c}_{1}, \ldots, \boldsymbol{c}_{k}\right]
\end{aligned}
$$

And let $\boldsymbol{G}^{2}$ and $\Theta^{2}$ be the counterpart of $\boldsymbol{G}^{1}$ and $\Theta^{1}$ respectively, which are associated with Tx2.

$$
\begin{aligned}
& \boldsymbol{S}= {\left[\begin{array}{cc}
\boldsymbol{G}^{1} \Theta^{1} & \boldsymbol{G}^{2} \Theta^{2} \\
\boldsymbol{G}^{2^{*}} \Theta^{2} & -\boldsymbol{G}^{1^{*}} \Theta^{l}
\end{array}\right] } \\
& \boldsymbol{R}_{z}= \boldsymbol{S} \boldsymbol{S}^{\prime}+\sigma_{n}^{2} \boldsymbol{I} \\
& \boldsymbol{R}_{z b l}= {\left[\begin{array}{cc}
\boldsymbol{G}^{1} \boldsymbol{c}_{1}^{1} & \boldsymbol{G}^{2} \boldsymbol{c}_{1}^{2} \\
\boldsymbol{G}^{2^{*}} \boldsymbol{c}_{1}^{2} & -\boldsymbol{G}^{l^{*}} \boldsymbol{c}_{1}^{l}
\end{array}\right] } \\
& \boldsymbol{W}_{\boldsymbol{I}}=\boldsymbol{R}_{z}^{-1} \boldsymbol{R}_{z b l}
\end{aligned}
$$

By applying $\boldsymbol{W}_{\boldsymbol{I}}$ on $\boldsymbol{Y}(n)$;

$$
\boldsymbol{D}_{\text {MMSE (1) }}=\boldsymbol{W}_{\boldsymbol{1}}^{\prime} \times \boldsymbol{Y}(n)
$$

The output of the MMSE detector is employed to Viterbi decoder for decoding STTC data. This decoded data is then compared with original transmitted sequence $\boldsymbol{a}_{k}(n)$ for finding bit error rate (BER) [17,18].

\section{Simulation Results}

The simulation is done for STBC MC-CDMA systems, STTC MC-CDMA systems, and STTC-STBC MCCDMA systems for 4,8 , and 16 states with different combinations of diversity for $K=5$ users. The user symbols are drawn from a unit-energy BPSK (binary phase shift keying) constellation. Walsh-Hadamard codes with processing gain $P=Q=32$ are used for spreading.

We assume a rich scattering environment and generate the FIR channel coefficients as i.i.d. complex Gaussian random variables with zero mean and variance $1 / \mathrm{M}$ [15]. The SNR (signal to noise ratio) is defined as $S N R=10 \log _{10} \frac{1}{\sigma_{n}^{2}}$ in $\mathrm{dB}$.

In Table 1 we present the bit error rate (BER) of 
MMSE detector for different states of STTC-MC-CDMA systems versus the SNR in a Rayleigh fading environment for $K=5$ users and 10000 bit sequences. 100 independent trials are taken for each SNR and their average is plotted. This table is also shows the BER of two standard single-user signals combining schemes (MRC and EGC) for comparison. From Table 1, it is concluded that the MMSE detector outperforms the single-user signals combining schemes in known channel. This is due to the reason that EGC/MRC is basically single user detection techniques and is not able to effectively suppress the MAI as compared to MMSE detector. Here, we have also studied the BER performance of the MMSE detector for the STTC-MC-CDMA system with the different states of STTC-MC-CDMA systems.

Table 2 show the performance of STBC MC-CDMA systems, STTC MC-CDMA systems, and STTC-STBC MC-CDMA systems for diversity $2 \times 1$ employing MMSE detector. Simulations results are also shown in Figures 2, 3 and $\mathbf{4}$ for MIMO antenna systems. With all these simulations it is noted that the performance gain can be enhanced up to around $5 \mathrm{~dB}$ at $10^{-3}$ BER by increasing the diversity from $2 \times 1$ to $2 \times 3$ in 16 states STTC-STBC MC-CDMA systems with MMSE detector as shown in Figures 5, 6 and 7.

Table 1. Comparison with different detection techniques.

\begin{tabular}{|c|c|c|c|c|c|c|c|c|c|}
\hline \multirow{3}{*}{ SNR } & \multicolumn{9}{|c|}{ BER } \\
\hline & \multicolumn{3}{|c|}{$\begin{array}{l}\text { STTC-MC-CDMA } \\
\text { Using MRC Detection }\end{array}$} & \multicolumn{3}{|c|}{$\begin{array}{l}\text { STTC-MC-CDMA } \\
\text { Using EGC Detection }\end{array}$} & \multicolumn{3}{|c|}{$\begin{array}{c}\text { STTC-MC-CDMA } \\
\text { Using MMSE Detection }\end{array}$} \\
\hline & 4-State & 8-State & 16-State & 4-State & 8-State & 16-State & 4-State & 8-State & 16-State \\
\hline 0 & 0.3568 & 0.3743 & 0.3882 & 0.2552 & 0.3097 & 0.4053 & 0.3101 & 0.3648 & 0.3978 \\
\hline 2 & 0.3255 & 0.3262 & 0.3389 & 0.1802 & 0.2143 & 0.3454 & 0.2448 & 0.2849 & 0.3236 \\
\hline 4 & 0.2967 & 0.2748 & 0.2703 & 0.1025 & 0.1260 & 0.2649 & 0.1618 & 0.1764 & 0.2072 \\
\hline 6 & 0.2692 & 0.2246 & 0.1993 & 0.0497 & 0.0541 & 0.1712 & 0.0756 & 0.0706 & 0.0823 \\
\hline 8 & 0.2533 & 0.1777 & 0.1412 & 0.0171 & 0.0180 & 0.0901 & 0.0208 & 0.0159 & 0.0170 \\
\hline 10 & 0.2390 & 0.1432 & 0.0996 & 0.0043 & 0.0036 & 0.0402 & 0.0025 & 0.0014 & 0.0015 \\
\hline
\end{tabular}

Table 2. Comparison with different coding schemes.

\begin{tabular}{cccc}
\hline \multirow{2}{*}{ SNR } & \multicolumn{2}{c}{ BER Using MMSE Detection } \\
\cline { 2 - 4 } & STBC-MC-CDMA & STTC-MC-CDMA & STTC-STBC-MC-CDMA \\
\cline { 2 - 4 } $\mathbf{0}$ & 0.0606 & 0.2680 & 0.0684 \\
$\mathbf{2}$ & 0.0191 & 0.1853 & 0.0174 \\
$\mathbf{4}$ & 0.0025 & 0.0996 & 0.0020 \\
\hline
\end{tabular}

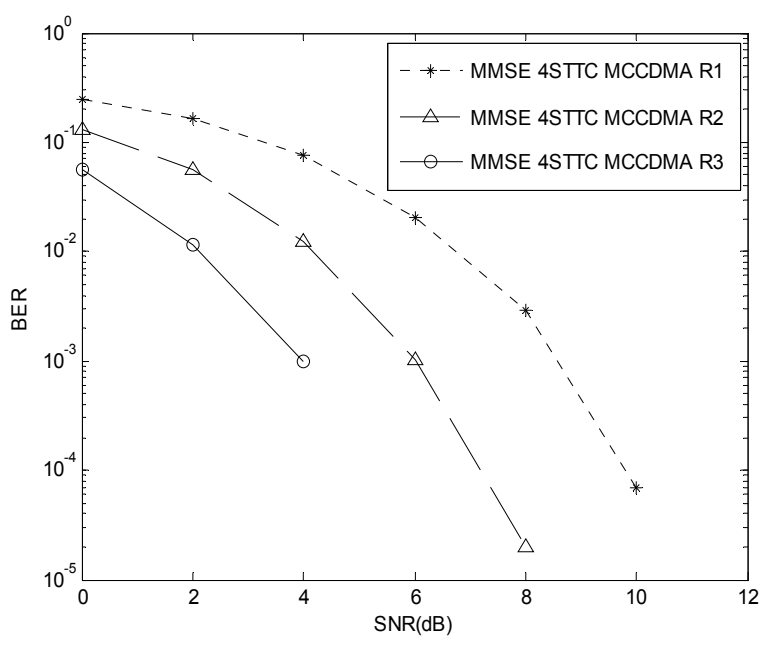

Figure 2. BER performance for 4 states STTC-MC-CDMA system using $2 \mathrm{Tx}$ and $1,2, \& \mathrm{Rx}$ antennas.

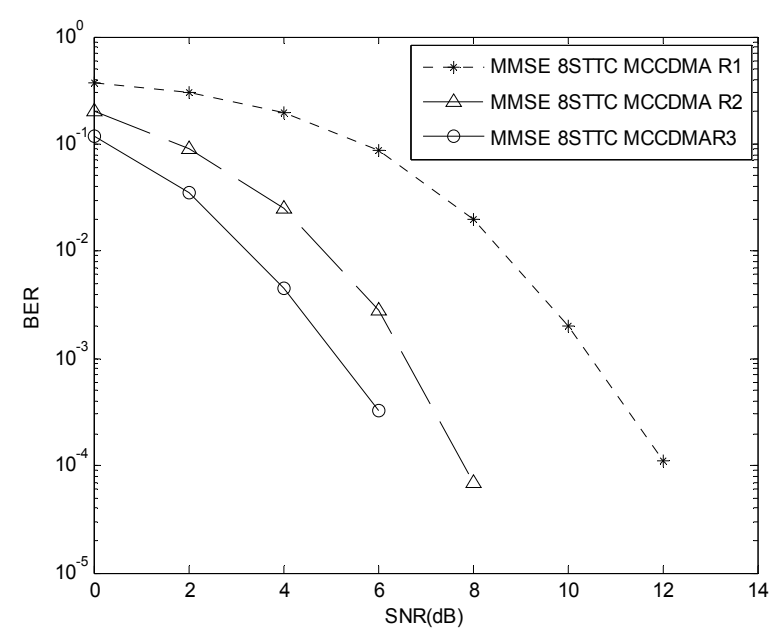

Figure 3. BER performance for 8 states STTC-MC-CDMA system using $2 \mathrm{Tx}$ and $1,2, \& 3 \mathrm{Rx}$ antennas. 


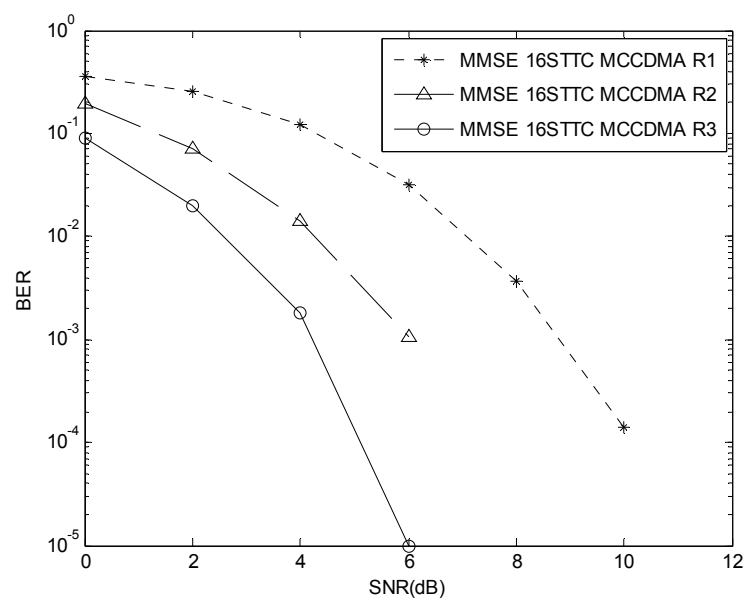

Figure 4. BER performance for 16 states STTC-MC-CDMA system using $2 \mathrm{Tx}$ and $1,2, \& 3 \mathrm{Rx}$ antennas.

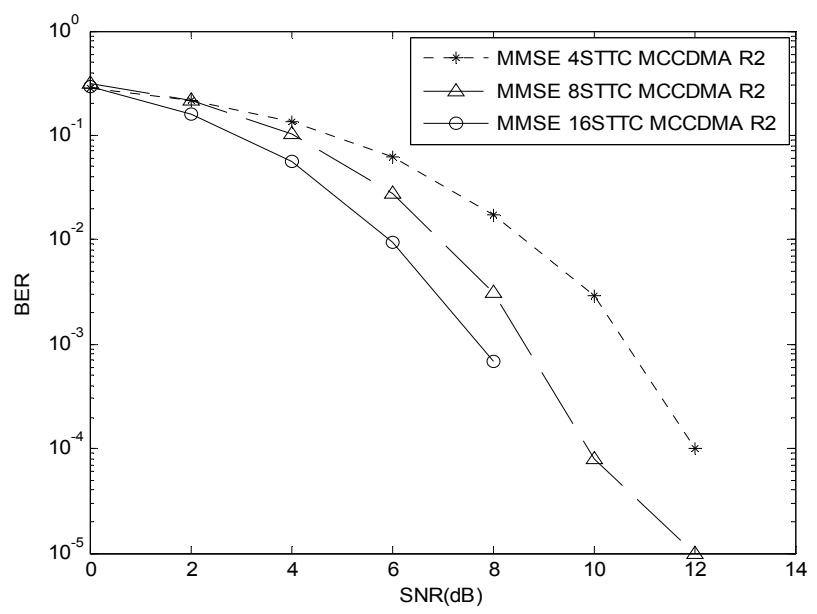

Figure 5. BER performance for $4,8, \& 16$ states STTC-MCCDMA system using $2 \mathrm{Tx}$ and $2 \mathrm{Rx}$ antennas.

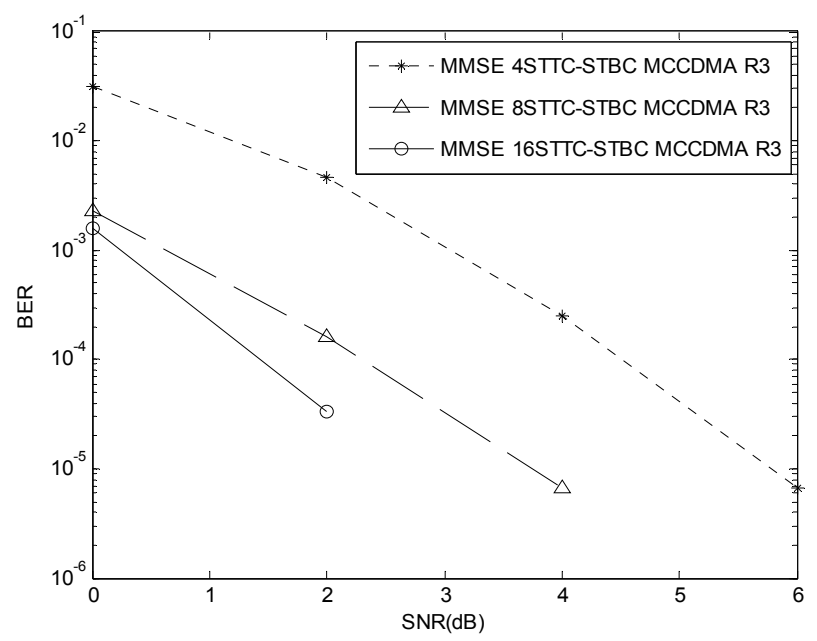

Figure 6. BER performance for $4,8, \& 16$ states STTCSTBC MC-CDMA system using 2 Tx and $3 \mathrm{Rx}$ antennas.

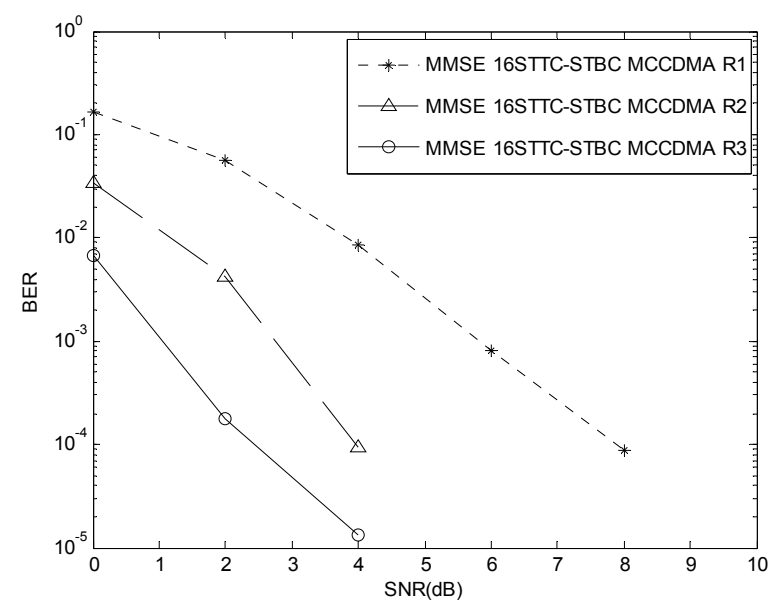

Figure 7. BER performance of 16 states STTC-STBC MCCDMA system using $2 \mathrm{Tx}$ and $1,2, \& 3 \mathrm{Rx}$ antennas.

\subsection{Computational Complexity Calculations}

Computational complexity in decoding is an important issue. Viterbi algorithm is used to decode the actual trellis codeword. The numeric computational complexity involved is calculated as follows.

Consider $L=2^{v}$ is the number of states where $v$ is the number of delay elements in the STTC encoder. The number of operations for multiplication/division are $\left\{n_{R}\left(n_{T}+1\right) 2^{L}\right\}$, comparisons are $\left\{2^{L}-1\right\}$, and additions/ subtractions are $\left\{\left[n_{R}\left(n_{T}+1\right)-1\right] 2^{L}\right\}$. It may be noted that $n_{T}$ is the number of transmitting and $n_{R}$ is the number of receiving antennas.

For example the numeric computational complexity for 4 states, 2 transmitter and 2 receiver antennas are:

Multiplication/Division are

$$
\begin{aligned}
& =n_{R}\left(n_{T}+1\right) 2^{L}=96 ; \\
\text { Comparisons are } & =2^{L}-1=15 ;
\end{aligned}
$$

$\&$ Addition/Subtraction are

$$
=\left[n_{R}\left(n_{T}+1\right)-1\right] 2^{L}=80
$$

Compare to one receive antenna the no. of multiplication/division operations involved are two times (as evident from Equation (20)). However, the improvement in the performance is approximately $2 \mathrm{~dB}$ at $10^{-3}$ probability of error employing MMSE detection as shown in the simulation results. This demonstrates the tradeoff involved in computational complexity and performance improvement.

\section{Conclusions}

In this paper, BER performance for STBC-MC-CDMA 
systems, STTC-MC-CDMA systems, and STTC-STBC MC-CDMA systems is evaluated using simulation for different states in known channel environment. For signal detection MMSE, MRC, and EGC receivers are considered. The simulation results are presented for the evaluation of the multi transmit multi receive antenna systems employing Viterbi decoding.

It is noted that the performance of STTC-STBC MC-CDMA system using MMSE detector is better than other schemes considered in this paper. Performance of all the schemes improves with the increase in number of states and antenna diversity. In particular, it is observed that STTC-STBC MC-CDMA systems using MMSE detector gives advantage of around $5 \mathrm{~dB}$ in terms of SNR while increasing diversity from $2 \times 1$ to $2 \times 3,16$ states and at $10^{-3}$ BER. This improvement in performance is noted at the cost of increased computational complexity which is calculated and mentioned in this paper.

\section{References}

[1] V. Tarokh, N. Seshadri and A. R. Calderbank, "SpaceTime Codes for High Data rate Wireless Communication: Performance Criterion and Code Construction," IEEE Transactions on Information Theory, Vol. 44, No. 2, March 1998, pp. 744-765.

[2] H. Sampath, S. Talwar and J. Tellado, "A Fourth Generation MIMO-OFDM Broadband Wireless System: Design, Performance, and Field Trial Results," IEEE Communication Magazine, September 2002, pp. 143-149.

[3] M. Juntti, M. Vehkapera, J. Leinonen, "MIMO MC-CDMA Communications for Future Cellular Systems," IEEE Communication Magazine, Feburary 2005, pp. 118-124.

[4] S. Hijazi, B. Natarajan and Z. Wu., "Flexible Spectrum use and Better Coexistence at the Physical Layer of Future Wireless Systems via a Multicarrier Platform," IEEE Wireless Communication, April 2004, pp. 64-71.

[5] E. A. Sourour and Masao Nakagawa, "Performance of Orthogonal Multicarrier CDMA in a Multipath Fading Channel," IEEE Transactions on Communications, Vol. 44, No. 3, March 1996, pp. 356-366.

[6] S. Hara and R. Prasad, "An Overview of Multi-Carrier CDMA," IEEE Communication Magazine, Vol. 35, No. 12, December 1997, pp. 126-133.

[7] S. L. Miller and B. J. Rainbolt, "MMSE Detection of Multicarrier CDMA," IEEE Journal on Selected Areas in Communications, Vol. 18, No. 11, November 2000, pp. 2356-2362.

[8] K. K. Wong, R. D. Murch and K. B. Letaief, "Perform ance Enhancement of Multiuser MIMO Wireless Communication Systems," IEEE Transactions on Communications, Vol. 50, No. 12, December 2002, pp. 1960-1968.

[9] A. F. Naguib, V. Tarokh, N. Seshadri and A. R. Calderbank, "A Space-Time Coding Modem for High-Data-Rate Wireless Communications," IEEE Journal on Selected Areas in Communication, Vol. 16, No. 8, October 1998, pp. 1459-1478.

[10] S. M. Alamouti, "A Simple Transmit Diversity Technique for Wireless Communications," IEEE Journal on Selected Areas in Communications, Vol. 16, No. 8, October 1998, pp. 1451-1458.

[11] A. Trivedi and M. Bansal, "Adaptive Equalization of Space-Time Coded MC-CDMA Systems," Communication, Computers and Signal Processing, IEEE Pacific Rim, 22-24 August 2007, pp. 186-189.

[12] W. Sun, H. B. Li and M. Amin, "A Subspace-Based Channel Identification Algorithm for Forward Link in Space-Time Coded MC-CDMA Systems," IEEE Proceedings, 2002, pp. 445-448.

[13] F. S. Ostuni, M. R. Nakhai and H. Aghvami, "Iterative Multi-User MMSE Receiver for Space-Time Trellis Coded CDMA over Frequency Selective Channels," IEEE 14th International Symposium on Personal, Indore and Mobile Radio Communication Proceedings, Chennai 2003, pp. 1968-1972.

[14] D. Mottier, D. Castelain, J. F. Helard and J. Y. Baudais, "Optimum and Sub-Optimum Linear MMSE Multi-User Detection for Multi-Carrier CDMA Transmission Systems," IEEE Proceedings, 2001, pp. 868-872.

[15] Wei Sun, Hongbin Li and Moeness Amin, "MMSE Detection for Space-Time Coded MC-CDMA," IEEE International Conference on Communications, Cape Town, Vol. 5, No. 11-15, May 2003, pp. 3452-3456.

[16] L. A. P. Hernandez and M. G. Otero, "A New STB-TCM Coded MC-CDMA Systems with MMSE-SVA Based Decoding and Soft-Interference Cancellation," IEEE Proceedings, 2005, pp. 113-116.

[17] A. Trivedi and L. K. Bansal, "Performance Study of Space-Time Trellis Coded MC-CDMA System Employing Different Detection Techniques," IEEE Proceedings, World Championship Sports Network, Conducted by Indian Institute of Information Technology, Allahabad, December 27-29, 2008, pp. 137-140.

[18] A. Trivedi and L. K. Bansal, "Comparative Study of Space-Time Trellis Code Concatenated with Space-Time Block Code MC-CDMA System," IEEE Proceedings, International Anti Corruption Conference, Conducted by Thapar University, Patiala, March 6-7, 2009, pp. 1099-1102. 